\title{
THEORY OF CEPHEID PULSATION: EXCITATION MECHANISMS
}

\author{
John P. Cox \\ Joint Institute for Laboratory Astrophysics, University \\ of Colorado and National Bureau of Standards, Boulder, Co \\ 80309
}

Abstract. The various excitation mechanisms (elght in al1) that have been proposed to account for the vibrational instability of variable stars, are surveyed. The most widely applied one is perhaps the "envelope ionization mechanism." This can account for most of the essential characteristics of the "instability strip." A simple explanation of the period-luminosity relation of classical Cepheids is given. A few outstanding problems in pulsation theory are also listed.

\section{INTRODUCTION}

The main purpose of this paper is to survey the various excitation mechanisms which have been proposed down through the years to account for the observed light and radial velocity variations of Cepheids and of other types of variable stars, with particular emphasis on Cephelds themselves.

Historically, the main driving agent for the (essentially radial) pulsations of classical Cepheids was the first to be investigated, and this early investigation began about 30 years ago. Yours truly was heavily involved with this work. At the time, he was highly astonished at the paucity of research papers dealing with this seemingly fundamental problem. There were plenty of papers concerned mainly with details of light and velocity curves and the like. But almost no papers attacking the main problem of what drives the pulsations! The only ones that he recalls now were two papers by Eddington, published in the early 1940's (Eddington 1941, 1942). These papers had a large effect on the author's subsequent work. He also remembers early papers by Zhevakin and Krogdah1.

This early work culminated in two fundamental papers: one, by Baker \& Kippenhahn (1962), on Delta Cephei itself; the other, by Cox (1963), on simplified Cepheid envelope models. The work of Baker and Kippenhahn was later extended and refined (Baker \& Kippenhahn 1965). The term "kappa-mechanism" (see $\$ 3$ ) was first explicitly introduced by Baker \& Kippenhahn (1962). Definitive and reliable calculations of linear, radial, nonadiabatic pulsations of stellar models first became possible with use of a computer code originated and developed by Castor (1971). 
One of the important conclusions of this early work was that the pulsation amplitude in the deep stellar interior was very small. It was, in fact, far too small for the nuclear reactions, which supply the mean luminosity of the star, to have anything directly to do with the pulsations. This conclusion was based largely on an early study by Epstein (1950). It turns out that Cepheid pulsation is primarily an envelope phenomenon; the cause of such pulsations had to be sought in factors affecting these outer layers. The basic reason for the smallness of the pulsation amplitude in the deep stellar interior is ultimately that, according to current ideas, Cepheids are in an advanced stage of evolution and are consequently highly centrally concentrated.

The extension of these results into the nonlinear domain was first effected by Christy (1966) and by Cox, Cox, Olsen, King, and Eilers (1966). The latter authors introduced explicitly the term "gammamechanism" (see $\$ 3$ ).

Linear, nonradial pulsations of stars, in adiabatic theory, were first explored by Smeyers (1966) and Dziembowski (1971). (Reviews of the properties of nonradial stellar pulsations can be found in Ledoux \& Walraven 1958; Ledoux 1974; Cox 1976, 1980; Unno, Osaki, Ando \& Shibahashi 1979.) Extension of the theory into the nonadiabatic domain was effected by Ando \& Osaki (1975) (however, they neglected the Eulerian perturbations of gravitational potential, i.e., made the Cowling approximation), Salo \& Cox (1980), and perhaps others. A new technique for treating linear, nonradial, nonadiabatic oscillations of spherical stars has been developed by Pesnell (1984a). This technique uses Lagrangian, rather than Eulerian, variations, and possesses many advantages over previous schemes. It appears highly promising, and may even constitute a breakthrough in the field.

Attempts to treat convection in pulsating stars were carried out by a number of investigators. The complications introduced by convection are by no means trivial, and were ignored in all the above works. Some earlier unpublished work on this problem was carried out by Gough, which ultimately resulted in the study by Baker \& Gough (1979). The problem has also been investigated by, among others, Castor (1968); Deupree (1977a,b,c,d; 1980); Saio (1980); Gonczi (1981, 1982); Gonczi \& Osak1 (1980); Durney (1984); and Stellingwerf (1982a,b; 1984a,b,c). The most ambitious work is that of Deupree (1977a); but the papers by Stellingwerf are also impressive. Both of the latter authors are in essential agreement. However, in contrast to Deupree (1977a), Stellingwerf (1984a) found that convection had a slight effect on the blue edge of the instability strip.

The mechanism by which convection in the envelope can result in a red edge to the instability strip, was first identified by Deupree (1977a). This mechanism is explained in $\$ 3$ below. 


\section{GENERAL PRINCIPLES OF EXCITATION MECHANISMS}

In general, an excitation mechanism consists of a way, or mechanism, for transforming some other kind of energy into mechanical energy of stellar pulsations. The most clearcut example would be the transformation of some of the steady radiant energy flowing through the outer layers of a Cepheid variable, into pulsation energy. This latter energy may be estimated, for a typical Cepheid, to be $\sim 10^{41-43}$ ergs. We may take as the (steady) luminosity $\sim 10^{3 /}$ ergs $s^{-1}\left(\sim 10^{4} L_{0}\right.$, $\mathrm{L}_{\Theta}=$ solar luminosity). If only a hundred thousandth $\left(10^{-5}\right)$ of this energy is available for such a transformation, the entire pulsation energy of such a Cepheid could be supplied in only $\sim 10^{9}-10^{11} \mathrm{~s} \sim$ $10^{2-4}$ yr or $\sim 10^{3-5}$ pulsation periods.

As another example, consider the recently discovered (Kurtz 1982) "rapidly oscillating Ap stars." The total energy (kinetic) Involved in the observed oscillations is perhaps $\sim 10^{34}$ ergs or larger. On the other hand, the total (kinetic) energy involved in such a star's outer convection zone may be of the same order of magnitude or, more likely, one or two orders of magnitude larger. At any rate, there seems to be enough energy residing in such a star's outer convection zone, that if only a small fraction of this energy were transformed into oscillatory energy, there would be enough to account for Kurtz's observations.

\section{A SURVEY OF SPECIFIC EXCITATION MECHANISMS}

In our survey of specific excitation mechanisms that have been proposed, we shall consider the following:

(1) "Envelope Ionization Mechanisms." Crucial elements: $\mathrm{He}^{+}$, He, H, C, O. The kappa- and gamma-mechanisms. The $r$ (radius)-mechanism.

(2) Stellingwerf "Bump" Mechanism.

(3) Epsilon ( $\varepsilon$ )-Mechanism.

(4) Kato Overstable Convection (associated with a mean molecular weight gradient).

(5) Shibahashi Overstable Magnetic Convection (associated with a magnetic field).

(6) Osaki Overstable Convection (associated with rotation).

(7) Tidally Forced Oscillations (Kato).

(8) Kelvin-Helmholtz Instability (Ando).

\section{1 "Envelope ionization mechanisms"}

This kind of mechanism was first suggested by Eddington

(1941, 1942). However, he had the idea that hydrogen was the crucial element for classical Cepheids; this element was subsequently identified as $\mathrm{He}^{+}$, once-ionized helium (Zhevakin 1953; Cox 1980, Chap. 10). Eddington also thought that Cepheid pulsations basically were "driven" by nuclear reactions (the " $\varepsilon$-mechanism," see below) (King \& Cox 1968), and that the hydrogen ionization zone only served to reduce the damping.

The main purpose of such an "envelope ionization mechanism" is to furnish heat to these outer layers upon compression, or to release heat upon expansion. Nuclear energy sources can play no important role. 
Therefore, the above all-important gains and losses of heat must be accomplished by a modulation of the radiation flowing through these layers. This is a rather bizarre means by terrestrial standards: It is the leakage of heat that is varied in a stellar heat engine of the kind we are describing. On the other hand, it is the input of heat that is varied in a conventional internal combustion engine.

The basic destabilizing effect of an "envelope ionization mechanism" can be understood as follows. Any destabilizing agent operating in the outer stellar layers will be most effective if it is located in or near the approprlate "transition region." This region is that level above which the pulsations are extremely nonadiabatic and the energy flux is effectively "frozen-in" (because the material in these outermost regions has so little heat capacity), and below which the pulsations are almost adiabatic (quasi-adiabatic) (see Fig. l, adapted from Osaki 1982). In the "quasi-adiabatic interior" any "driving" is almost exactly cancelled by a nearly equal amount of damping, so that there is essentially no net driving in this region. In the "nonadiabatic exterior" there can be no driving because there is no modulation of the flux. In or near the "transition region" itself there can be driving immediately below this region; however, immediately above this region the damping might be eliminated by nonadiabatic effects, leaving only driving. This transition region can be roughly located in any star by the order-of-magnitude condition that the total internal energy in the layers above this region be of the same order as the energy radiated by the star (equilibrium luminosity L) in one pulsation period $\Pi$. Quantitatively, this statement may be expressed by the equation:

$$
\frac{\left\langle\mathrm{c}_{V} \mathrm{~T}^{\mathrm{T}}\right\rangle \mathrm{m}_{*}}{\mathrm{LI}} \sim 1
$$

where $m_{*}$ is the total mass lying above the transition region, $c_{V}$ is the specific heat at constant volume, $T_{*}$ is the temperature in the transition region, and angular brackets denote an appropriate average over these outer layers. Although equation (1) was derived for radial

Fig. 1. Schematic drawing of the transition region. Adapted from Osaki (1982).

\section{SURFACE}

$\left.\begin{array}{l}\text { FLUX VARIATIONS } \\ \text { "FROZEN-IN:" VERY } \\ \text { NON-ADIABATIC }\end{array}\right\}$ NO DRIVING

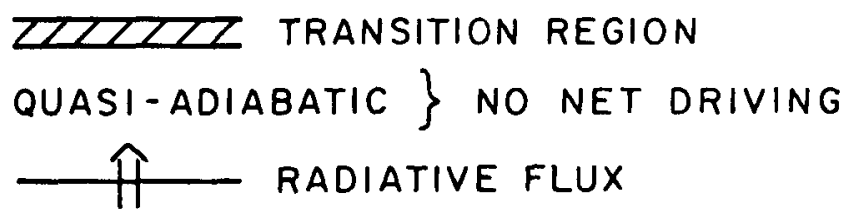


oscillations, it has recently been shown (Pesnell 1984b) that this equation also applies, to order of magnitude, to nonradial oscillations whose $\ell$ value is not too large, where $\ell$ is the latitudinal "quantum number" of a spherical harmonic. Further discussion of equation (1) may be found in Pesnell (1984b).

An "ionizing element" (a crucial element in the mid-stages of lonization) will, if this element is sufficiently abundant, produce a destabilizing effect essentially by causing the local temperature to be lower than if ionization were not occurring. This lonization represents additional internal degrees of freedom, so that any energy added to the gas (e.g., the energy supplied by adlabatic compression) goes partly into these additional degrees of freedom rather than into the kinetic energy of thermal motions. Hence, for example, such a gas will remain relatively cool upon compression. Malcolm Savedoff once expressed this relative coolness upon compression rather vividly as "putting ice cubes" into the appropriate stellar layers when they are most compressed. This relative coolness upon compression, say, will have further consequences, as follows.

The local opacity will now increase upon compression, whereas, normally, it decreases upon compression. The basic reason for this behavior is that the opacity normally varles about as the inverse cube of the local temperature, which is now remaining more-or-less constant because of the ionization. This statement is still qualitatively true even in the hydrogen fonization region of the $\mathrm{ZZ}$ Cet1 (white dwarf) stars, where the destabilization is supposed to be occurring (see below). A figure in Winget (1981) shows that, in the regions of interest, the opacity decreases, for given density, with increasing temperature. On the other hand, this opacity is roughly proportional to the density, which is still increasing upon compression. These two behaviors explain the "kappa-mechanism" (Baker \& Kippenhahn 1962), which refers to an increase of the opacity upon compression.

This increase of the local opacity upon compression will tend to "trap" the energy inside trying to get out. This "trapped" energy will instead get absorbed by the gas, and, through nonadiabatic effects, cause the pressure upon subsequent expansion to be larger than if the pulsations had been adiabatic. In this way any inclpient pulsations will tend to get "pumped up." Such a region is therefore destabilizing.

Similarly, the relative coolness of layers undergolng compression will cause these layers to radiate less. This diminished radiation upon compression has been termed the "gamma-mechanism" (Cox, Cox, 01sen, King \& Eilers 1966). The overall effect will be the same as for the kappa-mechanism, and will be a destabilizing tendency.

Finally, upon compression the total radiating area of the star will be reduced. This effect will also tend to "trap" radiation inside the star. Such an effect has been termed the " $r$ (radius)-effect" (Baker 1966), and will also contribute to instability. 
These considerations permit us to see rather easily how convection in the envelope can "quench" pulsations and result in a "red edge" of the instability strip (Deupree 1977a). One of Deupree's important findings was that convective transfer is most efficient when the star is most compressed. Hence, near minimum stellar radius convection causes the rate of energy loss from the star to be near maximum. This is exactly the condition for damping; this condition is just the opposite of that for driving. One may say that convection essentially "undoes" the trapping of energy effected by the kappa-, gamma-, and $r$-mechanisms discussed above. These last two mechanisms are still operating, even when convection in the envelope is present. However, their combined driving effect is small compared to the above damping effect of convection.

Envelope Ionization mechanisms have recently been invoked to explain successfully the observed nonradial oscillations of the variable white dwarfs, the "ZZ Ceti" stars (Winget et al. 1982a). Only, in this case, the crucial element is apparently hydrogen (see Winget \& Fontaine 1982 for a good review of the $\mathrm{ZZ}$ Ceti stars).

These considerations have been extended to the DB, or hellum, white dwarfs (Winget et al. 1982b). In this case, the crucial element is apparently He (neutral hellum, not once-ionized helium, as for the classical Cepheids). For the first time, a prediction as to the variability of the DB white dwarfs was made (Winget et al. 1982a), and subsequently a DB variable was actually detected observationally (this was GD 358) (Winget et al. 1982b, 1983). Three more have since been found (Winget et al. 1984, Winget 1984); these are PG $1654+160$, PG $1116+158$, and $P G 1351+489$.

It has recently been suggested (Starrfield, Cox \& Hodson 1981, 1983; Starrfleld, Cox, Kidman \& Pesnell 1984) that an envelope ionization mechanism, with carbon and/or oxygen as the crucial element(s), may account for the instability of the very hot variable PG 1159-035 (see also Winget, Hansen \& Van Horn 1983).

\subsection{Stellingwerf "bump" mechanism}

The "bump" mechanism was suggested by Stellingwerf (1978, 1979) and was applied in Stellingwerf (1978) and in Cox \& Stellingwerf (1979) to the Beta Cephei stars. This may be considered a kind of "envelope Ionization mechanism," although, as we shall see below, its operation does not really depend on ionization.

It is well known that the effectiveness of any destabilizing mechanism operating in regions of a star where the energy is carried predominantly or solely by radiation is very sensitive to certain features of the opacity. One of these features is the temperature dependence of the opacity law. The Stellingwerf "bump" mechanism can be effective because it introduces a "wiggle," or "bump," on the opacity versus temperature curve, for given density. Such a "bump" has a region where the opacity decreases with increasing temperature less rapidly 
than immediately above or below this region (i.e., the opacity vs. temperature curve has a relatively "flat" region) (see Fig. 2). This feature causes such a region to radiate less strongly than the regions immediately above or below it, when the material is compressed. Such a region can therefore impede the flow of radiation through these layers, in somewhat the same way as the kappa-, gamma-, and r-mechanisms operate.

Roughly, the bump is, essentially, a result of the coincidence of the peak of the Planck function with the ionization edge of $\mathrm{He}^{+}$(at $54.4 \mathrm{eV}$ ). The bump accordingly occurs at a temperature close to $1.5 \times$ $10^{5} \mathrm{~K}$, and is rather insensitive to density. It turns out that the temperature of the "transition region" in Beta Cephei stars is close to this value (Cox 1967, p. 90), so it might be expected that this bump might have something to do with the Instability of these stars (Cox \& Stellingwerf 1979). In this study it was also shown that radiation pressure, in only modest amounts, could increase cv quite significantly. However, in a very careful study by Lee \& Osaki (1982) it was shown that this bump is not actually pronounced enough to cause instability in these stars. This "bump" mechanism is also discussed by Pesne11 (1983), who reaches the same conclusion as Lee \& Osaki (1982).

Fig. 2. Schematic opacity $(K)$ versus temperature $(T)$ curve, at fixed density $(\rho)$, showing the Stellingwerf "bump."

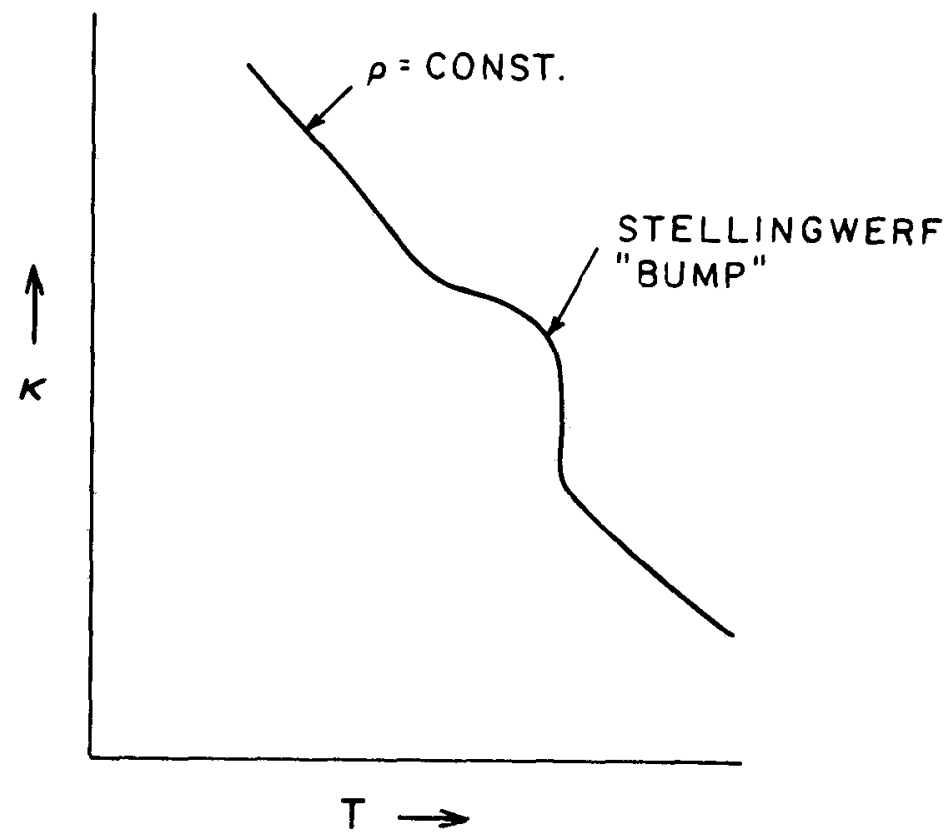




\subsection{Epsilon $(\varepsilon)$-mechanism}

The $\varepsilon$-mechanism is the excitation effect of thermonuclear reactions. It essentially always (at least in principle) exerts a destabilizing tendency, although the effects of this mechanism on actual variable stars are usually negligible (Cox 1967).

The destabilizing tendency arising from the $\varepsilon$-mechanism derives from several factors. First, the rate of energy production per unit mass $\varepsilon$ from thermonuclear reactions is generally given to adequate accuracy by the approximate interpolation formula

$$
\varepsilon \propto \rho^{\lambda} T^{\nu}
$$

Here $\lambda$ is usually 1 or 2 but $\nu$ may be quite large (e.g., $v \approx 15-17$ for the carbon cycle, $v \approx 4$ for the proton chains, and $v \sim 30-40$ for helium-burning reactions). Second, in regions where nuclear reactions are occurring the oscillations are usually nearly adiabatic, so that, for example,

$$
\frac{\delta T}{T}=\left(\Gamma_{3}-1\right) \frac{\delta p}{\rho},
$$

where a " $\delta$ " denotes the Lagrangian variation and $\Gamma_{3}$ is one of the adiabatic exponents. Hence, usually,

$$
\frac{\delta \varepsilon}{\varepsilon} \approx\left[\lambda+\nu\left(\Gamma_{3}-1\right)\right] \frac{\delta \rho}{\rho},
$$

where the quantity in square brackets can be rather large.

What this means is that, upon compression, a relatively large amount of thermonuclear energy may be generated. If this energy is not carried away, it may be absorbed by the local matter, enhance the local temperature increase, and so lead to overstable oscillations.

The agent that may carry this excess energy away is radiation. If we consider an "element" which has been slowly displaced inward in a region where the material is "subadiabatic" (i.e., convectively stable according to the Schwarzschild criterion), the element will be warmer than its local surroundings, and so will radiate more copiously than they (of course, an outward-displaced element will be cooler than its local surroundings, and so will radiate less copiously than they). Whether this excess energy will be carried away (or whether the deficit of energy will be eliminated by heat gains from the surroundings) depends on the size of the element under consideration. The total amount of energy lost (or gained) by radiation in a given time will be proportional to the surface area of the element, and hence to the square of 1 ts size. On the other hand, the total excess (or deficit) of thermonuclear energy generated in this same time will be proportional to the volume of the element, and hence to the cube of its size. Hence large elements are more likely, from this effect, to cause overstability than small elements.

Note that, if the temperature gradient in the relevant regions were superadiabatic rather than subadiabatic (yet such that the elements 
are convectively stable according to the Ledoux criterion), the roles of nuclear energy production and radiative transfer would be reversed. In this case a rising element, for example, would be hotter than its surroundings. The enhanced loss of heat from the element (as compared to its surroundings) due to radiation would now tend to lead to overstability (Kato effect, Kato 1966; also, see $\$ 3.4$ ), whereas the enhanced gain of heat due to an increased thermonuclear reaction rate would now tend to lead to damping.

An overstability in hydrogen-burning and helium-burning shells in evolved stars (in such stars the temperature gradient in the relevant regions was always subadiabatic) has recently been investigated by Kippenhahn (1983). He finds that, generally, hydrogen-burning shells are too thin ever to be overstable. However, such an overstability can sometimes exist in helium-burning shells. In particular, he finds that such shells can be overstable during portions of "thermal pulses," when the evolutionary track of the star "loops" for a time to much higher effective temperatures. What the observational consequences of this overstability are, are not clear (see Kippenhahn 1983).

In immediately post-main sequence stars it is known that there is a thin hydrogen-burning shell which supplies most of the energy radiated by the star. Moreover, in this shell the mean molecular weight $\mu$ increases inward from a value appropriate to predominantly hydrogen in the exterior to a value appropriate to nearly pure helium in the core. In this thin region there is, arising from the $\mu$-gradient, an additional contribution to the restoring force acting on a displaced mass element (see $\$ 3.4$ ). Consequently, the Brunt-Väisälä frequency here is relatively large. One can therefore have "propagating" g modes, or spatially oscillatory g modes, in this region, with these modes "evanescent" above and below, as was first shown by Osaki (1976). He referred to this property as a "trapping" of modes. In addition, their amplitudes may be relatively large here, and their vertical wavelengths small, as was also shown by Osaki (1976). Even so, he finds that the driving resulting from $E$-mechanism here was insufficient to destabilize the entire star. The fact is, the radiative dissipation in this region is very large, so large that this damping completely predominates over the nuclear driving. This large radiative damping is essentially a consequence of the very short wavelengths of these trapped modes. In a sense, short wavelengths are analogous to too-small elements in the discussion of the Kippenhahn overstability above. Thus, the concluston was that the $\varepsilon$-mechanism operating in the hydrogenburning shell of slightly post-main sequence stars could not account for the pulsations of the Beta Cephei stars, as had been suggested earlier by Chiosi (1974) and by Aizenman, Cox \& Lesh (1975).

\subsection{Kato overstable convection}

In a region in a star where a mean molecular welght

gradient is present, it is possible, as was first pointed out by Kato (1966), that overstable convection can exist. This kind of overstability might occur in a region that is convectively stable according to the "Ledoux criterion," i.e., an element of matter displaced slowly 
upward, for example, would have a greater density than 1ts surroundings, and so would tend to sink. In the absence of nonadiabatic effects, such an element would simply oscillate indefinitely up and down at the local Brunt-Väisälä frequency.

What these nonadiabatic effects will do, however, depends on the local average temperature gradient. If this temperature gradient is subadiabatic, such an upward-displaced element will be cooler than its surroundings. It will therefore absorb heat from them during its upward swing. This absorption of heat will decrease the density of the element below the value appropriate for adiabatic motion, and so will cause its subsequent descent to be slower than for adiabatic motion. In this way it can be seen that the oscillatory motion of the element will eventually be damped out by these nonadiabatic effects.

On the other hand, if the average temperature gradient is superadiabatic, then a slowly rising element will be warmer than its surroundings, and so w11l lose heat to them. This loss of heat during the upward swing will lead to a further enhancement of the density of the element, and so it will subsequently sink faster than if it had lost no heat. Similarly, a sinking element will subsequently rise faster than in the adiabatic case. In this case it can be seen that the amplitude of this oscillatory motion will gradually increase, as a result of these nonadiabatic effects. This phenomenon of an oscillatory motion of a mass element with a gradually growing amplitude is known as overstable convection.

It is clear, moreover, that overstable convection can only occur when the mean molecular weight $\mu$ increases toward the center of the star. But this is, after all, the normal situation encountered in stellar evolution. We assume that the motion of the convective element is so slow that pressure equilibrium with its surroundings will have been established. Then, if a rising element is to have a net downward buoyant force, 1ts density must be greater than that of its surroundings. Hence, the quantity $(T / \mu)(T=$ temperature $)$ of the element must be less than that of its surroundings. The value of $\mu$ for the element is characteristic of its level of origin. If $\mu$ increases inward, it is clear that the temperature of the element could be greater than that of its surroundings (the condition for overstability), and the element could still be convectively stable (i.e., the net buoyant force on an upward-displaced element would be downward).

Note that the Kato overstable convection mechanism can also be looked at as follows. The $\mu$-gradient is equivalent to an additional contribution to the total force acting on a displaced mass element computed as if the chemical composition were uniform. This additional contribution to the force is proportional to the factor $-(d \ln \mu / d \ln P) \delta \mathrm{r}$, where $P$ denotes total pressure and $\delta r$ is the (radial) displacement of a mass element from its equilibrium position. Thus, if $d$ ln $\mu / d$ \&n $P>0$ (the normal situation in stellar evolution), we have: if $\delta r>0$ (the element is displaced upward), this additional force is downward, and acts as an extra contribution to the restoring force. If $\overline{\delta r<0}$ 
(downward displacement), this additional force is upward, and thus in this case too it increases the restoring force. Moreover, this additional contribution to the restoring force may be considered constant with time, since this force will change only on a nuclear time.

Now, if the average temperature gradient is superadiabat1c, the buoyant force on an upward-displaced mass element will be upward, In the same direction as the displacement. In the absence of a $\mu-g r a d i e n t$ such a region would then be convectively unstable. However, the $\mu$ gradient may supply enough of a downward contribution to the restoring force acting on an upward-displaced element that the motion may still be oscillatory. But a rising element in a region of the star with a superadiabatic temperature gradient will be hotter than its surroundings, and so will lose heat to them. It can thus be seen that these heat exchanges will increase the density of an upward-displaced element, and so cause the buoyant force slowly to get weaker with time. On the other hand, the force arising from the $\mu$-gradient is remaining essentially constant with time. Thus the net restoring force (buoyant force plus the " $\mu$-gradient force") is gradually getting stronger with time. This situation of an oscillator with a slowly strengthening spring constant clearly corresponds to overstability.

These considerations will be helpful in connection with the mechanism to be discussed next, the "overstable magnetic convection," mechanism. In the semi-convective zone of a massive star we have all the necessary ingredients for the Kato overstable convection mechanism to operate. The convective elements are likely, moreover, to be rather massive (perhaps a tenth or a hundredth of a solar mass?). The 1dea therefore arose that, if such an overstability occurred with the convective elements in the convective core of a massive star, some kind of instability might well arise. This instability might concelvably account for the pulsations of the Beta Cephel stars. Such a suggestion has, in fact, been made by Percy (1970) and Spiegel (1970). However, the calculations of Shibahashi and Osak1 (1976) showed that, while this overstability was indeed present in the relevant regions, it was too weak to destabilize the whole star. The conclusion is that this mechanism will probably not account for the pulsations of the Beta Cephei stars nor of any other known kind of pulsating star.

\subsection{Shibahashi overstable magnetic convection}

The suggestion of "magnetic overstability" was made by Shibahashi (1983) to account for the observed oscillations ( $6-14 \mathrm{~min}$ ) in the recently discovered "rapidly oscillating Ap stars" (Kurtz 1982). A more physical discussion is given in Cox (1984).

It has been known for some time (e.g., Chandrasekhar 1952) that a convectively unstable region (e.g., the outer hydrogen convection zone) in the presence of only a moderately strong magnetic field can lead to overstable oscillations.

The mechanism by which overstable oscillations can arise in the present circumstances can be understood as follows. It is well known 
(e.g., Alfvén \& Fälthammer 1963) that magnetic lines of force "embedded" in an astrophysical plasma behave in many respects as if they were material, elastic strings "glued" to the plasma. Moreover, these lines of magnetic force exert a tension along their length, and so try to remain straight. On the other hand, convective motions will tend to produce "wiggles" in these lines of force. It can easily be seen, then, that this magnetic tension will amount to an additional contribution to the restoring force on a displaced mass element. This additional contribution to the restoring force will tend to induce oscillatory motion in an otherwise convectively unstable region; in this way a magnetic field will tend to suppress convection.

It is the exchange of heat (nonadiabatic effects) that leads to overstability, just as in the Kato overstability mechanism. Consider a superadiabatic region in a star. In such a region the buoyant force is in the same direction as the displacement. Therefore, such a region would be convectively unstable in the absence of a magnetic field. A rising mass element, for example, in such a region would, however, be hotter than its surroundings and so would lose heat to them. Hence the buoyant force would gradually get weaker with time. However, the additional contribution to the restoring force, being magnetic in origin (and proportional to the factor $-\left(\mathrm{B}^{2} / \mathrm{d}^{2}\right) \delta \mathrm{r}$, where $\mathrm{B}$ is the magnetic induction and $d$ is the diameter of the element), will change much more slowly in time (Cox 1984). The net restoring force (buoyant plus magnetic) on the element is thus slowly getting larger in time, and this corresponds to overstability. (In this sense "magnetic overstablifty" and the Kato overstable convection mechanism have much in common.) This mechanism and the Kato overstable convection mechanism may be considered as examples of the thermally excited oscillator of Moore and Spiegel (1966).

The growth times of the overstability can be shown to be fairly short, say less than a year. Moreover, the oscillatory periods should be only of the order of minutes (Cox 1984).

Whether this mechanism will continue to be a viable explanation of the observed variations of the "rapidly oscillating Ap stars," remains to be seen.

\subsection{Osaki overstable convection}

This mechanism was proposed by Osaki (1974) in order to attempt to find an excitation mechanism for the Beta Cephei stars. It is based on the known fact that rotation of a convective region in a star will introduce, via the Coriolis forces, an oscillatory component into the otherwise dynamically unstable motions. In other words, a rising or descending element will, because of the Coriolis force, swerve sideways and hence w111 execute a "circular" rather than an "up-and-down" motion. The period of this oscillatory motion will be determined by the rotation period of the convective region. The amplitude of this "circular" motion will increase at a rate determined by the magnitude of the superadiabaticity of the temperature gradient in the convective region. 
Thus, the convective elements in such a rotating convective region execute overstable oscillations. Note that this overstability has nothing to do with nonadiabatic effects; it would exist even if the oscillations were strictly adiabatic.

Osaki assumed that the convective region was the convective core of a massive star. If the rotation period of the core was properly chosen, this overstable convection might resonate with the nonradial $f$ mode of the radiative envelope (this mode has a period in the proper range for these stars). Thus, the whole star might be rendered unstable, and the Beta Cephei phenomenon might be accounted for.

This idea is very ingenious, and the parameter values required for it to work properly are certainly reasonable. The trouble is, however, that in nature all the requirements may not be satisfied, and this mechanism has not been generally accepted as an explanation of the instability of the Beta Cephei stars.

\subsection{Tidally forced osclilations}

This mechanism was suggested by Kato (1974) in an attempt to account for the often-observed double periodicity in Beta Cephei stars. Specifically, he pointed out that if the frequency separation between two rotationally split "m-sublevels" of a rotating star is very close to the orbital frequency of a companion, a resonance might occur which could amplify one (or more) of these sublevels. However, such a resonant interaction can occur only if a mode is already excited by some other mechanism. The paper is perhaps not fundamental in the sense that it offers no suggestion as to what this other mechanism might be.

There are many interesting points raised in this paper. A number of these resonant interactions require special circumstances, which may or may not be satisfied in nature. Still, many of the points raised in this paper should be kept in mind by those working in the field.

\subsection{Kelvin-Helmholtz instability}

This mechanism was suggested by Ando (1981) again in an attempt to account for the instability of the Beta Cephei stars. The Instability basically derives from a Kelvin-Helmholtz instability in the thin region between a supposed rapidly spinning core and a slower spinning envelope. In this thin region there is strong shear, which may be subject to a Kelvin-Helmholtz instability (Papaloizou \& Pringle 1978). There is also here, incidentally, a large $\mu$-gradient. The frequency of this Kelvin-Helmholtz instability may, moreover, be almost the same as that of one of the $f$ or $g$ nonradial modes of the rest of the star. If this is the case, a resonance might occur which might excite one or more of these modes. In this way the excitation of some of the observed nonradial oscillation modes of the Beta Cephe1 stars and of the "line profile varlable B stars" ("53 Per" stars) (Smith 1978, 1980a,b; Smith \& McCall 1978; Smith \& Buta 1979) might be accounted for. 
The author points out a number of strong points in favor of the present mechanism for the kinds of stars cited above. He also notes that this mechanism is not as restrictive as the rotating convective core mechanism of Osaki (1974) (see earlier).

The final word on this mechanism has not yet been said. The mechanism looks promising, and only time will tell how successful it is.

\section{APPLICATION TO CEPHEIDS}

The $\mathrm{He}^{+}$envelope Ionization mechanism was applied to Cepheid variables by Baker \& Kippenhahn (1962) and Cox (1963). One of the most important aspects of this work (verified many times both previously and subsequently) is that it showed the following: It is the approximate colncidence of the "transition region" and the region of 50 percent lonization of $\mathrm{He}^{+}$(both in the envelope) that defines the "Cepheid instability strip" on the Hertzsprung-Russell (H-R) diagram. This strip cuts through the classical Cepheids, the $W$ Virginis variables, the BL Herculis stars (KIng, Cox \& Hodson 1981; Hodson, Cox, \& KIng 1982; Cox \& Wallersteln 1984), the RR Lyrae variables, the Delta Scuti stars, the Ap stars, and may even extend down to the white dwarfs (see F1g. 3).

These works and many subsequent calculations have confirmed the somewhat ubiquitous period-mean density relation of pulsating stars:

Fig. 3. Schematic Hertzsprung-Russell diagram, showing instability strip.

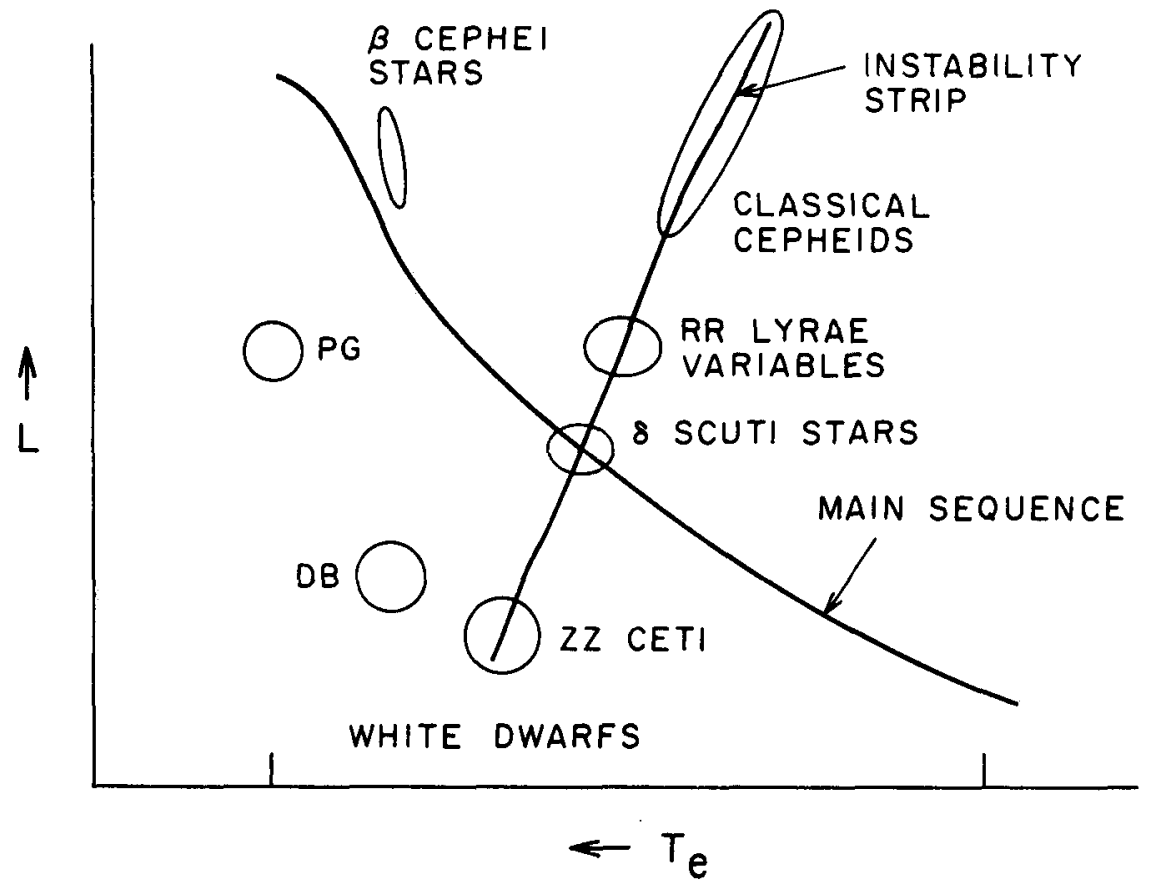




$$
\text { II } \sqrt{\bar{\rho}} \approx \text { const }
$$

for a given mode, where $I$ denotes the perfod of the mode and $\bar{\rho}$ is the mean density of the star. Hence small, compact stars generally have shorter perfods than do large, tenuous stars. This relation even holds, approximately, for nonradial oscillations of not-too-high orders or degrees (at least for $p$ modes).

One feature of the above instability strip, along with the period-mean density relation, make the period-luminosity relation of Cepheids particularly easy to understand. (This relation has been confirmed many times, both observationally and theoretica1ly.) This feature is that the strip is almost vertical on an H-R diagram. In turn, the reason for this feature is that, for a star of given mean luminosity, colncidence of the transition region and the region of 50 percent ionization of $\mathrm{He}^{+}$occurs for a sharply defined value of the effective temperature. Moreover, this value does not depend strongly on other stellar parameters. Thus, all stars in the strip have roughly the same effective temperatures. Hence, approximately,

$$
L \propto R^{2}
$$

for such stars, where $R$ is the mean radius of the star and $L$ is its mean luminosity. Thus, large stars in the strip (those in its upper regions) are more luminous than smaller stars. On the other hand, according to the perlod-mean density relation,

$$
\pi \propto \mathrm{R}^{3 / 2} / \mathrm{M}^{1 / 2},
$$

approximately, where $M$ is the mass of the star. Eliminating $R$ between equations (6) and (7) gives

$$
\frac{L}{M^{2 / 3}} \propto \pi^{4 / 3} .
$$

For $M=$ constant, we have

$$
\mathrm{L} \propto \Pi^{4 / 3}
$$

For $L \propto M^{4}$, we get

$$
\mathrm{L} \propto \Pi^{8 / 5}
$$

The empirical relation is approximately

$$
\mathrm{L} \propto \Pi \quad \text {. }
$$

The rough relations (9) and (10) are not in terribly bad agreement with the empirical relation (11), considering the crudity of the derivation. In the simplest terms, one may say that the more luminous stars in the instability strip are large, and large stars have longer periods than do small stars. Hence one might expect a positive correlation to exist between period and luminosity for stars in the instability strip. 


\section{SOME OUTSTANDING UNSOLVED PROBLEMS}

I suppose a review of Cepheid theory and of excitation mechanisms in pulsating stars would not be complete without at least a listing of current outstanding unsolved problems. Such a list, as seen by this reviewer, is presented in this section.

\subsection{Time-dependent convection}

Great strides have recently been made in incorporating treatments, in some approximation or other, of time-dependent convection into pulsating star calculations. We now even have a theoretical red edge to the instability strip, thanks largely to the work of Deupree $(1977 \mathrm{a})$. As shown by the rather impressive set of papers by Stellingwerf $(1982, a, b ; 1984 a, b, c)$, a treatment of time-dependent convection that is not too complicated can now be conveniently put into codes for the computation of radial oscillations of stars. This last work not only agrees satisfactorily with that of Deupree, but it also shows that convection may play an important role in the problem of the limiting amplitudes of pulsating stars. It would be useful if some not-too-complicated scheme for treating time-dependent convection, somewhat analogous to Stellingwerf's for radial oscillations, could be developed for use in connection with nonradial oscillations.

In spite of these successes, the general problem of the interaction of time-dependent convection with pulsations cannot be considered as solved. There is st 111 considerable need for rather fundamental studies along these lines, such as that of Toomre and collaborators (see the review paper by Toomre 1982). As has been emphasized by Toomre (1982), the treatments referred to in the preceding paragraph still involve a number of somewhat uncomfortably arbitrary stellar parameters. There is still a great need for more fundamental work on the problem (e.g., Toomre, Hurlburt \& Massaguer 1984).

\subsection{Cepheid masses}

Securing agreement between masses of pulsating stars as inferred, on the one hand, from evolution theory, and, on the other, from pulsation theory, is still a problem. Perhaps most severe are the "beat masses" of double-mode classical Cepheids. (These are the masses of double-mode pulsators derived from the first overtone fundamental perfod ratio and the fundamental period itself.) Despite the low values of these masses, the actual masses of these stars are probably normal, i.e., as expected from evolution theory (e.g., Cox 1982). Why is pulsation theory leading to such low mass values for these stars?

It is also puzzling that beat masses for RR Lyrae variables are not anomalously low. Both of these problems are discussed by A. N. Cox in an important review paper (Cox 1982 and references therein). Perhaps the augmented metal opacities idea of Simon $(1982 a, b)$ has some merit, despite certain rather strong objections (Huebner \& Magee 1984). 


\subsection{Cepheid "bumps"}

The physical explanation of the "bumps" which often appear on the descending portions of the light curves for Cepheids with periods in the approximate range 7-11 days (the "Hertzsprung relation," Hertzsprung 1926) is not yet avallable. These "bumps," when they occur, usually also show up in the approprlate velocity curves. The "bumps" were attributed by Christy (1967) to an "echo" phenomenon. In this process a shock wave was generated in the stellar envelope, travelled to the stellar center, was reflected, and subsequently travelled again to the surface, and produced the "bump." On the other hand, it was suggested by Simon \& Schmidt (1976) that the "bumps" were the result of a resonance condition, in which the second overtone (radial) period was almost exactly one-half of the fundamental perlod.

At first sight, these two explanations appear quite different and independent of each other. However, as Simon has said (quoted from Whitney 1982), "...the pulse and resonance models may merely be opposite sides of a single coin." This possibility has been explored in a preliminary way by Whitney (1982), and further elaborated on by Alkawa \& Whitney (1984). The results of Buchler (1984) are also consistent with this idea.

Petersen $(1981,1984)$ has noted that Population II Cephelds also exhibit "bumps" whose phase of occurrence depends on period, analogously to the Hertzsprung relation in classical Cepheids.

\subsection{Double mode cepheids}

As yet, Initial-value type stellar pulsation calculations have not succeeded in producing true double-mode behavior (1.e., a long-lasting admixture of both fundamental and first overtone pulsation modes). The physical cause of double-mode behavior continues to elude us. An excellent and very comprehensive review (with lots of references!) has been published by A. Cox (1982). I think I can add nothing to what has been said here. As has been emphasized in this last reference, the stars which exhibit double-mode behavior probably have normal masses for their luminosities (i.e., as given by evolution theory). One would like to know not only what causes this kind of behavior, but also why the ratio of first overtone to fundamental periods is so low for the classical Cepheids.

\subsection{The excitation mechanism for Beta Cephel stars}

The cause of the pulsations of the Beta Cephel star's remains as one of the outstanding problems of pulsation theory. This problem has persisted for more than three-quarters of a century, despite many attempts at solution.

The most recent review article on possible excitation mechanisms for these stars is the excellent one by Osaki (1982). Earlier reviews are by Kato (1976) and $\operatorname{Cox}(1976)$.

The most recent mechanism is that suggested by Ando (1981), based on a Kelvin-Helmholtz instability in a thin shear layer surrounding a 
rapidly spinning core. While not as restrictive as the rapidly spinning convective core model of Osaki (1974), still special assumptions are required with this model.

\subsection{Miscellaneous problems}

Besides the above problems, there are the following rather general ones: (1) the R Coronae Borealis stars specifically, and very nonadiabatic stellar pulsations generally. (2) The Mira variables specifically, and long period variables generally; here time-dependent convection might have important applications; also, the relation between pulsations and mass loss should be investigated. (3) The very hot star PG 1159-035 and others of its kind; here the excitation mechanism should be clarified; temporal changes due to stellar evolution are probably important. (4) The general problem of the relation between magnetic flelds and stellar pulsations should be further investigated. (5) The complicated nature of neutron star oscillations should be studied further. (6) The nature and cause of solar oscillations, and what they can tell us about the solar interior (helioseismology), should be studied more. (7) Here I put all other problems which I have not thought of and which are not listed above.

\section{SUMMARY}

In this paper we have surveyed the main destabilizing mechanisms (eight in al1) that have been proposed to account for the instability of pulsating stars of known types. Perhaps the most widely applied type of mechanism is the "envelope ionization mechanism." This mechanism can account for most of the essential characteristics of the "Instability strip" (e.g., Cox 1980, p.146). A simple interpretation of the period-luminosity relation of classical Cepheids is given. Finally, we list a few outstanding problems in pulsation theory.

We are grateful for helpful conversations with Art Cox, Carl J. Hansen, W. Dean Pesne11, Bernhard Durney, and Don Winget. Many thanks for preprints, etc., to $\mathrm{H}$. Ando, Robert Buchler, Art Cox, Bernhard Durney, George Gonczl, Carl Hansen, Icko Iben, Phil Marcus, Barbara \& Dimitri Mihalas, Chander Mohan, W. Dean Pesne11, Jorgen Petersen, H. Saio, H. Shibahashi, Norman Simon, Joe Smak, Paul Smeyers, Myron Smith, Sumner Starrfield, Bob Stellingwerf, Mine Takeuti, Juri Toomre, Craig Wheeler, Chuck Whitney, Lee Anne Willson, Don Winget and many others. We also thank Dean Pesnell and Carl Hansen for reading and commenting on the manuscript. This work was supported in part by National Science Foundation grant No. AST83-15698 through the University of Colorado.

\section{REFERENCES}

Aikawa, T. \& Whitney, C. A. (1984). Astrophys. J., in press. Atzenman, M. L., Cox, J. P. \& Lesh, J. R. (1975). Astrophys. J., 197, 399.

Alfvén, H. \& Fälthammer, C. G. (1963). Cosmical Electrodynamics. Oxford: Clarendon Press.

Ando, H. (1981). M.N.R.A.S., 197, 1139. 
Ando, H. \& Osaki, Y. (1975). Publ. Astron. Soc. Japan, 27, 581. Baker, N. (1966). In Stellar Evolution, eds. R. F. Stein \& A. G. W. Cameron, p. 333. New York: Plenum Press.

Baker, N. \& Gough, D. O. (1979). Astrophys. J., 234, 232.

Baker, N. \& Kippenhahn, R. (1962). Z. Astrophys., 54, 114.

Baker, N. \& Kippenhahn, R. (1965). Astrophys. J., 142, 868.

Buchler, R. (1984). Private communication.

Castor, J. I. (1968). Unpublished manuscript.

Castor, J. I. (1971). Astrophys. J., 166, 109.

Chandrasekhar, S. (1952). Ph11. Mag., Ser. 7, 43, 501.

Chiosi, C. (1974). Astron. Astrophys., 37, 281.

Christy, R. F. (1966). Astrophys. J., $\frac{144}{144} 108$.

Christy, R. F. (1967). In Aerodynamic Phenomena in Stellar Atmospheres, ed. R. N. Thomas, p. 105. New York: Academic.

Cox, A. N. (1982). In Pulsations in Classical and Cataclysmic Variable

Stars, eds. J. P. Cox \& C. J. Hansen, p. 157. Boulder: JILA.

Cox, A. N. \& Wallerstein, G. (1984). Preprint.

Cox, J. P. (1963). Astrophys. J., 138, 487.

Cox, J. P. (1967). In Aerodynamic Phenomena in Stellar Atmospheres,

ed. R. N. Thomas, p. 3. New York: Academic Press.

Cox, J. P. (1976). Ann. Rev. Astron. Astrophys., 14, 247.

Cox, J. P. (1976). In Proc. Solar and Stellar Pulsation Conf., eds.

A. N. $\overline{C o x}$ and R. G. Deupree, p. 127. Los Alamos: LANL.

Cox, J. P. (1980). Theory of Stellar Pulsation. Princeton: Princeton

Univ. Press.

Cox, J. P. (1984). Astrophys. J., in press.

Cox, J. P., Cox, A. N., Olsen, K. H., King, D. S. \& Eilers, D. D.

(1966). Astrophys. J., 144, 1038.

Cox, J. P. \& Stellingwerf, R. F. (1979). Pub. A.S.P., 91, 319.

Deupree, R. G. (1977a). Astrophys. J., 211, 509.

Deupree, R. G. (1977b). Astrophys. J., 214, 502 .

Deupree, R. G. (1977c). Astrophys. J., 215, 232 .

Deupree, R. G. (1977d). Astrophys. J., 215, 620 .

Deupree, R. G. (1980). Astrophys. J., 236, 225.

Durney, B. (1984). Preprint.

Dzlembowski, W. (1971). Acta Astron., 21, 289.

Eddington, A. S. (1941). M.N.R.A.S., 101, 182.

Eddington, A. S. (1942). M.N.R.A.S., 102, 154 .

Epstein, I. (1950). Astrophys. J., 112,6 .

Gonczi, G. (1981). Astron. Astrophys., 96, 138.

Goncz1, G. (1982). In Pulsations in Classical and Cataclysmic Variable

Stars, eds. J. P. Cox \& C. J. Hansen, p. 206. Boulder:

JILA.

Goncz1, G. \& Osaki, Y. (1980). Astron. Astrophys., 84, 304 .

Hertzsprung, E. (1926). B.A.N., 3, 115 .

Hodson, S. W., Cox, A. N. \& King, D. S. (1982). Astrophys. J., 253, 260 .

Huebner, W. \& Magee, N. (1984). Preprint.

Kato, S. (1966). Publ. Astron. Soc. Japan, 18, 374.

Kato, S. (1974). Pub1. Astron. Soc. Japan, $\overline{26}, 341$.

Kato, S. (1976). In Multiple Periodic Variable Stars, ed. W. S. Fitch,

p. 3. Dordrecht: Reidel. 
King, D. S. \& Cox, J. P. (1968). Pub1. A.S.P., 80, 365.

King, D. S., Cox, A. N. \& Hodson, S. W. (1981). Astrophys. J., 244, 242.

Kippenhahn, R. (1983). Preprint.

Kurtz, D. W. (1982). M.N.R.A.S., 200, 807 .

Ledoux, P. (1974). in Stellar Instability and Evolution, eds. P.

Ledoux, A. Noels \& A. W. Rodgers, p. 135. Dordrecht:

Reidel.

Ledoux, P. \& Walraven, Th. (1958). Handbuch der Physik, ed. S. Flügge, 51, p. 353. Berlin: Springer-Verlag.

Lee, U. \& Osaki, Y. (1982). Publ. Astron. Soc. Japan, 34, 39.

Moore, D. W. \& Spiegel, E. A. (1966). Astrophys. J., 1ㄹ, 871 .

Osak1, Y. (1974). Astrophys. J., 189, 469.

Osaki, Y. (1976). Publ. Astron. Soc. Japan, 28, 105.

Osaki, Y. (1982). In Pulsations in Classical and Cataclysmic Variable

Stars, eds. J. P. Cox \& C. J. Hansen, p. 303. Boulder:

JILA.

Papaloizou, J. \& Pringle, J. E. (1978). M.N.R.A.S., 182, 423.

Percy, J. R. (1970). Astrophys. J., 159, 177.

Pesne11, W. D. (1983). Ph.D. Dissertation, University of Florida.

Pesnell, W. D. (1984a). Preprint.

Pesne11, W. D. (1984b). Astrophys. J., in press.

Petersen, J. 0. (1981). Astron. Astrophys., 96, 146.

Petersen, J. O. (1984). Preprint.

Saio, H. (1980). Astrophys. J., 240, 685.

Saio, H. \& Cox, J. P. (1980). Astrophys. J., 236, 549.

Shibahashi, H. (1983). Astrophys. J. (Lett.), 275, L5.

Shibahashi, H. \& Osak1, Y. (1976). Publ. Astron. Soc. Japan, 28, 199.

Simon, N. R. (1982a). In Pulsations in Classical and Cataclysmic

Varlable Stars, eds. J. P. Cox \& C. J. Hansen, p. 221.

Boulder: JILA.

Simon, N. R. (1982b). Astrophys. J. (Lett.), 260, L87.

Simon, N. R. \& Schmidt, E. G. (1976). Astrophys. J., 205, 162.

Smeyers, P. (1966). Acad. Roy. des Sci., des Lett. et des Beaux.-Arts d. Belgique, $5^{\mathrm{e}}$ Ser., 52, 1126 .

Smith, M. A. (1978). Astrophys. J., 224, 927 .

Smith, M. A. (1980a). In Nonlinear and Nonradial Oscillations of the Sun and Stars, eds. H. A. Hil1 \& W. Dziembowski, p. 60 . New York: Springer-Verlag.

Smith, M. A. (1980b). In Highlights of Astronomy, ed. P. A. Wayman, 5 , p. 457. Dordrecht: Reidel.

Smith, M. A. \& Buta, R. J. (1979). Astrophys. J. (Lett.), 232, L193.

Smith, M. A. \& McCal1, M. L. (1978). Astrophys. J., 223, 221 .

Spiegel, E. A. (1970). Comments on Astrophys. and Space Sci., 1, 57.

Starrfield, S. G., Cox, A. N. \& Hodson, S. W. (1981). Space Scí. Rev., 27, 621 .

Starrfield, S. G., Cox, A. N. \& Hodson, S. W. (1983). Astrophys. J. (Lett.), 268, L227.

Starrfield, S. G., Cox, A. N., Kidman, R. B. \& Pesne11, W. D. (1984). Astrophys. J., in press.

Stellingwerf, R. F. (1978). Astron. J., 83, 1184.

Stellingwerf, R. F. (1979). Astrophys. J., 227, 935. 
Stellingwerf, R. F. (1982a). Astrophys. J., 262, 330 .

Stellingwerf, R. F. (1982b). Astrophys. J., $\frac{262}{27}, 339$.

Stellingwerf, R. F. (1984a). Astrophys. J., $\overline{277}, 322$.

Stellingwerf, R. F. (1984b). Astrophys. J., 277, 327 .

Stellingwerf, R. F. (1984c). Preprint.

Toomre, J. (1982). In Pulsations in Classical and Cataclysmic Variable Stars, eds. J. P. Cox \& C. J. Hansen, p. 170. Boulder: JILA.

Toomre, J., Hurlburt, N. E. \& Massaguer, J. M. (1984). Preprint (to appear in Proc. Small-Scale Dynamical Processes in Quiet 